\title{
Plasma diagnostics of emission-line galaxies in SDSS
}

\author{
Zhitai Zhang ${ }^{1,2}$, Yanchun Liang ${ }^{1}$ and François Hammer ${ }^{3}$ \\ ${ }^{1}$ Key Laboratory of Optical Astronomy, National Astronomical Observatories, \\ Chinese Academy of Sciences, Beijing 100012, China \\ email: ztzhang@nao.cas.cn, ycliang@bao.ac.cn \\ ${ }^{2}$ University of Chinese Academy of Sciences, Beijing 100049, China \\ ${ }^{3}$ GEPI, Observatoire de Paris, Section de Meudon, 92195 Meudon, France
}

\begin{abstract}
Sample contains 15019 narrow emission-line galaxies, i.e. Seyferts, LINERs, composites and star-forming galaxies, from SDSS DR7 for systematically studying differences between different classes. We report two clear sequences of electron temperature $\left(T_{e}\right)$ and density $\left(n_{e}\right): T_{e-L I N E R} \gtrsim T_{e-\text { composite }}>T_{e-\text { Seyfert }}>T_{e-\text { star-forming }}$ and $n_{e-\text { Seyfert }} \gtrsim n_{e-L I N E R}>$

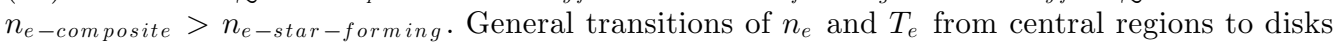
are quantitatively confirmed.
\end{abstract}

Keywords. galaxies: active, galaxies: ISM, galaxies: Seyfert, galaxies: starburst, surveys

\section{Brief Overview \& Quick Results}

Our emission-line galaxy sample is selected by requiring $\mathrm{S} / \mathrm{N}>5$ in strong emissionlines, and is divided into four classes through applying the galaxy classification scheme from Kewley et al. (2006). Plasma diagnostics are obtained through $I[\mathrm{~S}$ II $] \lambda 6716 / \lambda 6731$ and $I[\mathrm{O}$ III $] \lambda 5007 / \lambda 4363$ with simultaneous determination for $n_{e}\left[\mathrm{~S}\right.$ II] and $T_{e}[\mathrm{O}$ III] in 15 019 sampled galaxies. We further identify three groups according to the physical aperture size of SDSS 3-arcsec diameter fibers $\phi(\mathrm{kpc})$ and $F W H M\left(\mathrm{~km} \mathrm{~s}^{-1}\right)$ of $\mathrm{H} \alpha$ (Bennert et al. 2006; Kollatschny \& Wang 2006): FWHM > 300 for "NLR-dominated" (labeled ND; $\ln \phi$ $<1$ ) and "disk-contaminated NLR" (labeled $D C$; $\ln \phi>1$ ) objects; $F W H M<300$ for "non-NLR" objects (labeled $N N$ ). Table 1 summarizes our results. By comparing stellar mass, specific star formation rate and plasma diagnostic results, we propose that $Y_{L} \geqslant$ $Y_{S F}>Y_{C}>Y_{S F}$, where $Y$ is the characteristic present-day star-formation time scale. Moreover, in the case of AGNs, we find that several strong lines of evidence suggest some supplementary energy source(s) should be responsible for high ionization potential.

Acknowledgements. The authors thank the symposium organizers for their invitation to this poster. This work was supported by the Natural Science Foundation of China (NSFC) Foundation under Nos.10933001, 11273026.

Table 1. Summary of the mean values of $n_{e}$ and $T_{e}$.

\begin{tabular}{|c|c|c|c|c|c|c|c|c|c|c|c|c|}
\hline \multirow[b]{3}{*}{ Number } & \multicolumn{3}{|c|}{ Seyfert } & \multicolumn{3}{|c|}{ LINER } & \multicolumn{3}{|c|}{ composite } & \multicolumn{3}{|c|}{ Star-forming } \\
\hline & ND & DC & $\mathrm{NN}$ & ND & DC & NN & ND & DC & NN & ND & DC & $\mathrm{NN}$ \\
\hline & 89 & 1,052 & 701 & 7 & 75 & 13 & 35 & 348 & 955 & 3 & 165 & 11,576 \\
\hline$n_{e}\left[\mathrm{~cm}^{-3}\right]$ & 415 & 332 & 160 & 230 & 201 & 113 & 208 & 150 & 77 & 166 & 152 & 57 \\
\hline$T_{e}\left[10^{4} \mathrm{~K}\right]$ & 1.40 & 1.32 & 1.29 & 2.13 & 1.37 & 2.73 & 1.76 & 1.68 & 1.61 & 1.78 & 1.77 & 1.37 \\
\hline
\end{tabular}

\section{References}

Bennert, N., Jungwiert, B., Komossa, S., Haas, M., \& Chini, R. 2006, A\&̛A, 456, 953

Kewley, L. J., Groves, B., Kauffmann, G., \& Heckman, T. 2006, MNRAS, 372, 961

Kollatschny, W. \& Wang, T. G. 2008, ApESSS, 303, 123 